\title{
High-dimensional convex sets arising in algebraic geometry
}

\author{
Yanir A. Rubinstein
}

June 20, 2019

Dedicated to Bo Berndtsson on the occassion of his $68^{\text {th }}$ birthday

\begin{abstract}
We introduce an asymptotic notion of positivity in algebraic geometry that turns out to be related to some high-dimensional convex sets. The dimension of the convex sets grows with the number of birational operations. In the case of complex surfaces we explain how to associate a linear program to certain sequences of blow-ups and how to reduce verifying the asymptotic log positivity to checking feasibility of the program.
\end{abstract}

|

\section{Introduction}

Convex sets have long been known to appear in algberaic geometry. A well-known example whose origins can be traced to Newton and Minding are the convex polytopes associated to toric varieties [6, 8, 19], also known as Delzant polytopes in the symplectic geometry literature [3]. In recent years, this notion has been further extended to any projective variety, the so-called Newton-Okounkov bodies (or 'nobodies'). In the most basic level, avoiding a formal definition, such a body is a compact convex body (not necessarily a polytope) in $\mathbb{R}^{n}$ associated to two pieces of data: a nested sequence of subvarieties inside a projective variety of complex dimension $n$, and a line bundle over the variety. Among other things, beautiful relations between the notion of volume in algebraic geometry and the volume of these bodies have been proved [12,14.

The purpose of this note, motivated by a talk in the High-dimensional Seminar at Georgia Tech in Decemeber 2018, is to associate another type of convex bodies to projective varieties. The main novelty is that this time the convex bodies can have unbounded dimension while the projective variety has fixed dimension (which, for most of the discussion, will be in fact 2 (i.e., real dimension 4)). In fact, the asymptotic behavior of the bodies as the dimension grows (on the convex side) corresponds to increasingly complicated birational operations such as blow-ups (on the algebraic side). Rather than volume, we will be interested in intersection properties of these bodies. This gives the first relation between algebraic geometry and asymptotic convex geometry that we are aware of.

This note will be aimed at geometers on both sides of the story-convex and algebraic. Therefore, it will aim to recall at least some elementary notions on both sides. Clearly, a rather unsatisfactory compromise had to be made on how much background to provide, but it is our hope that at least the gist of the ideas are conveyed to experts on both sides of the story.

Research supported by NSF grant DMS-1515703. I am grateful to I. Cheltsov and J. Martinez-Garcia for collaboration over the years on these topics, to G. Livshyts for the invitation to speak in the High-dimensional Seminar in Georgia Tech, to the editors for the invitation to contribute to this volume, and to a referee for a careful reading and catching many typos. 


\section{$1.1 \quad$ Organization}

We start by introducing asymptotic log positity in $₫ 2$. It is a generalization of the notion of positivity of divisors in algebraic geometry, and the new idea is that it concerns pairs of divisors in a particular way. In $₫ 3$ we associate with this new notion of positivity a convex body, the body of ample angles. In \$4 we explain how two previously defined classes of varieties (asymptotically log Fano varieties and asymptotically log canonically polarized varieties) fit in with this picture. The problem of classifying two-dimensional asymptotically log Fano varieties has been posed in 2013 by Cheltsov and the author and is recalled (Problem 4.2) as well as the progress on it so far. In $\$ 5$ we make further progress on this problem by making a seemingly new connection between birational geometry and linear programming, in the process explaining how birational blow-up operations yield convex bodies of increasingly high dimension. Our main results, Theorems 5.5 and [5.6. first reduce the characterization of "tail blow-ups" (Definition 5.3) that preserve the asymptotic log Fano property to checking the feasibility of a certain linear program and, second, show that the linear program can be simplified. The proof, which is the heart of this note, involves associating a linear program to the sequence of blow-ups and characterizing when it is feasible. The canonically polarized case will be discussed elsewhere. A much more extensive classification of asymptotically log del Pezzo surfaces is the topic of a forthcoming work [16] and we refer the reader to Remark [5.10 for the relation between Theorems 5.5 and 5.6 and that work.

This note is dedicated to Bo Berndtsson, whose contributions to the modern understanding and applications of convexity and positivity on the one hand, and whose generosity, passion, curiosity, and wisdom on the other hand, have had a lasting and profound influence on the author over the years.

\section{Asymptotic log positivity}

The key new algebraic notion that gives birth to the convex bodies alluded to above is asymptotic log positivity. Before introducing this notion let us first pause to explain the classical notion of positivity, absolutely central to algebraic geometry, on which entire books have been written 13 .

\subsection{Positivity}

Consider a projective manifold $X$, i.e., a smooth complex manifold that can be embedded in some complex projective space $\mathbb{P}^{N}$. In algebraic geometry, one is often interested in notions of positivity. Incidentally, these notions are complex generalizations/analogues of notions of convexity. In discussing these notions one interchangeably switches between line bundles, divisors, and cohomology classe: 1 . Complex codimension 1 submanifolds of $X$ are locally defined by a single equation. Formal sums (with coefficients in $\mathbb{Z}$ ) of such submanifolds is a divisor (when the formal sums are taken with coefficients in $\mathbb{Q}$ or $\mathbb{R}$ this is called a $\mathbb{Q}$-divisor or a $\mathbb{R}$-divisor). By the Poincaré duality between homology and cohomology, a (homology class of a) divisor $D$ gives rise to a cohomology class $[D]$ in $H^{2}(M, \mathbb{F})$ with $\mathbb{F} \in\{\mathbb{Z}, \mathbb{Q}, \mathbb{R}\}$. On the other hand a line bundle is, roughly, a way to patch up local holomorphic functions on $X$ to a global object (a 'holomorphic section' of the bundle). The zero locus of such a section is then a formal sum of complex hypersurfaces, a divisor. E.g., the holomorphic sections of the

\footnotetext{
${ }^{1} \mathrm{~A}$ great place to read about this trinity is the cult classic text of Griffiths-Harris [7, §1.1] that was written when the latter was a graduate student of the former.
} 
hyperplane bundle in $\mathbb{P}^{N}$ are linear equations in the projective coordinates $\left[z_{0}: \ldots: z_{N}\right]$, whose associated divisors are the hyperplanes $\mathbb{P}^{N-1} \subset \mathbb{P}^{N}$. The associated cohomology class, denoted $[H]$, is the generator of $H^{2}\left(\mathbb{P}^{N}, \mathbb{Z}\right) \cong \mathbb{Z}$. The anticanonical bundle of $\mathbb{P}^{N}$, on the other hand, is represented by $[(N+1) H]$ and its holomorphic sections are homogeneous polynomials of degree $N+1$ in $z_{0}, \ldots, z_{N}$. Either way, both of these bundles are prototypes of positive ones, a notion we turn to describe.

Now perhaps the simplest way to define positivity, at least for a differential geometer, is to consider the cohomology class part of the story. A class $\Omega$ in $H^{2}(X, \mathbb{Z})$ admits a representative $\omega$ (written $\Omega=[\omega]$ ), a real 2 -form, that can be written locally as $\sqrt{-1} \sum_{i, j=1}^{n} g_{i j} d z^{i} \wedge \overline{d z^{j}}$ with $\left[g_{i j}\right]$ a positive Hermitian matrix, and $z_{1}, \ldots, z_{n}$ are local holomorphic coordinates on $X$. Since a cohomology class can be associated to both line bundles and divisors, this gives a definition of positivity for all three. As a matter of terminology one usually speaks of a divisor being 'ample', while a cohomology class is referred to as 'positive'. For line bundles one may use either word. A line bundle is called negative (the divisor 'anti-ample') if its dual is positive.

The beauty of positivity is that it can be defined in many equivalent ways. Starting instead with the line bundle $L$, we say $L$ is positive if it admits a smooth Hermitian metric $h$ with positive curvature 2-form $-\sqrt{-1} \partial \bar{\partial} \log h=: c_{1}(L, h)$. By Chern-Weil theory the cohomology class $c_{1}(L)=\left[c_{1}(L, h)\right]$ is independent of $h$.

\subsection{Asymptotic log positivity}

We define asymptotic log positivity/negativity similarly, but now we will consider pairs $(L, D)$ and allow for asymptotic corrections along a divisor $D$ (in algebraic geometry the word $\log$ usually refers to considering the extra data of a divisor). Let $D=D_{1}+\ldots+D_{r}$ be a divisor on $X$. We say that $\left(L, D=D_{1}+\ldots+D_{r}\right)$ is asymptotically log positive/negative if $L-\sum_{i=1}^{r}\left(1-\beta_{i}\right) D_{i}$ is positive/negative for all $\beta=\left(\beta_{1}, \ldots, \beta_{r}\right) \in U \subset(0,1)^{r}$ with $0 \in \bar{U}$. For the record, let us give a precise definition as well as two slight variants.

Definition 2.1. Let $L$ be a line bundle over a normal projective variety $X$, and let $D=$ $D_{1}+\ldots+D_{r}$ be a divisor, where $D_{i}, i=1, \ldots, r$ are distinct $\mathbb{Q}$-Cartier prime Weil divisors on $X$.

- We call $(L, D)$ asymptotically log positive/negative if $c_{1}(L)-\sum_{i=1}^{r}\left(1-\beta_{i}\right)\left[D_{i}\right]$ is positive/negative for all $\beta=\left(\beta_{1}, \ldots, \beta_{r}\right) \in U \subset(0,1)^{r}$ with $0 \in \bar{U}$.

- We say $(L, D)$ is strongly asymptotically log positive/negative if $c_{1}(L)-\sum_{i=1}^{r}\left(1-\beta_{i}\right)\left[D_{i}\right]$ is positive/negative for all $\beta=\left(\beta_{1}, \ldots, \beta_{r}\right) \in(0, \epsilon)^{r}$ for some $\epsilon>0$.

- We say $(L, D)$ is log positive/negative if $c_{1}(L)-[D]$ is positive/negative.

Note that log positivity implies strong asymptotic log positivity which implies asymptotic $\log$ positivity (ALP). None of the reverse implications hold, in general.

The usual notion of positivity can be recovered (by openness of the positivity property) if one required the $\beta_{i}$ to be close to 1 . By requiring the $\beta_{i}$ to hover instead near 0 we obtain a notion that is rather different, but more flexible and still recovers positivity. Indeed, asymptotic $\log$ positivity generalizes positivity, as $L$ is positive if and only if $\left(L, D_{1}\right)$ is asymptotically $\log$ positive where $D_{1}$ is a divisor associated to $L$. However, the ALP property allows us to 'break' $L$ into pieces and then put different weights along them, so that $(L, D)$ could be ALP even if $L$ itself is not positive. Let us give a simple example.

Example 2.2. Let $X$ be the blow-up of $\mathbb{P}^{2}$ at a point $p \in \mathbb{P}^{2}$. Let $f$ be a hyperplane containing $p$ and let $\pi^{-1}(f)$ denote the total transform (i.e., the pull-back), the union of two curves: the exceptional curve $Z_{1} \subset X$ and another curve $F \subset X$ (such that $\left.\pi\left(Z_{1}\right)=p, \pi(F)=f\right)$. 
Downstairs $f$ is ample, but $\pi^{-1}(f)$ fails to be positive along the exceptional curve $Z_{1}$. However, $\left(\pi^{-1}(f), Z_{1}\right)$ is ALP.

This example is not quite illustrative, though, since it is really encoded in a classical object in algebraic called the Seshadri constant. In fact in the example above one does not need to take $\beta$ small, rather it is really $1-\beta$ that is the 'small parameter' (and, actually, any $\beta \in(0,1)$ works, reflecting that the Seshadri constant is 1 here).

A better example is as follows.

Example 2.3. Let $X=\mathbb{F}_{n}$ be the $n$-th Hirzebruch surface, $n \in \mathbb{N}$. Let $-K_{X}$ be the anticanonical bundle. It is positive if and only if $n=0,1$. In general, $-K_{X}$ is linearly equivalent to the divisor $2 Z_{n}+(n+2) F$ where $Z_{n}$ is the unique $-n$-curve on $X$ (i.e., $Z_{n}^{2}=-n$ ) and $F$ is a fiber (i.e., $F^{2}=0$ ). A divisor of the form $a Z_{n}+b F$ is ample if and only if $b>n a$. Thus $\left(-K_{X}, Z_{n}\right)$ is ALP precisely for $\beta \in\left(0, \frac{2}{n}\right)$.

\section{The body of ample angles}

The one-dimensional convex body $\left(0, \frac{2}{n}\right)$ of Example 2.3 is the simplest that occurs in our theory. Let us define the bodies that are the topic of the present note.

Let $D=\sum_{i=1}^{r} D_{i}$, and denote

$$
L_{\beta, D}:=L-\sum_{i=1}^{r}\left(1-\beta_{i}\right) D_{i} .
$$

The problem of determining whether a given pair $\left(L, D=\sum_{i=1}^{r} D_{i}\right)$ is ALP amounts to determining whether the set

$$
\mathrm{AA}_{ \pm}(X, L, D):=\left\{\beta=\left(\beta_{1}, \ldots, \beta_{r}\right) \in(0,1)^{r}: \pm L_{\beta, D} \text { is ample }\right\}
$$

satisfies

$$
0 \in \overline{\mathrm{AA}_{ \pm}(X, L, D)}
$$

Thus, this set is a fundamental object in the study of asymptotic log positivity.

Definition 3.1. We call $\mathrm{AA}_{+}(X, L, D)$ the body of ample angles of $(X, L, D)$, and $\mathrm{AA}_{-}(X, L, D)$ the body of anti-ample angles of $(X, L, D)$.

Remark 3.2. The body of ample angles encodes both asymptotic log positivity and the classical notion of nefness. Indeed, if $(1, \ldots, 1) \in \overline{\mathrm{AA}_{ \pm}(X, L, D)}$ then $\pm L$ is numerically effective (nef).

Lemma 3.3. When nonempty, $\mathrm{AA}_{ \pm}(X, L, D)$ is an open convex body in $\mathbb{R}^{r}$.

Proof. Suppose $\mathrm{AA}_{+}(X, L, D)$ is nonempty. Openness is clear since positivity (and, hence, ampleness $)$ is an open condition on $H^{2}(X, \mathbb{R})$. For convexity, suppose that $\beta, \gamma \in \mathrm{AA}_{+}(X, L, D) \subset$ $\mathbb{R}^{r}$. Then, for any $t \in(0,1)$,

$$
\begin{aligned}
L_{t \beta+(1-t) \gamma, D} & =L-\sum_{i=1}^{r}\left(1-t \beta_{i}-(1-t) \gamma_{i}\right) D_{i} \\
& =(t+1-t) L-\sum_{i=1}^{r}\left(t+1-t-t \beta_{i}-(1-t) \gamma_{i}\right) D_{i} \\
& =t\left[L-\sum_{i=1}^{r}\left(1-\beta_{i}\right) D_{i}\right]+(1-t)\left[L-\sum_{i=1}^{r}\left(1-\gamma_{i}\right) D_{i}\right]
\end{aligned}
$$


is positive since the positive cone within $H^{2}(X, \mathbb{R})$ is convex. If $\beta, \gamma \in \mathrm{AA}_{-}(X, L, D) \subset \mathbb{R}^{r}$ we get

$$
-L_{t \beta+(1-t) \gamma, D}=t\left(-L_{\beta, D}\right)+(1-t)\left(-L_{\gamma, D}\right)
$$

so by the same reasoning $t \beta+(1-t) \gamma \in \mathrm{AA}_{-}(X, L, D)$.

Remark 3.4. One may wonder why we require $\mathrm{AA}(X, L, D)$ to be contained in the unit cube. Indeed, that is not an absolute must. However, we are most interested in the "small angle limit" as $\beta \rightarrow 0 \in \mathbb{R}^{r}$. Still, we require the coordinates to be positive (and not, say, limit to 0 from any orthant) since, geometrically, the $\beta_{i}$ can sometimes be interpreted as the cone angle associated to a certain class of Kähler edge metrics. One could in principle allow the whole positive orthant, still. But in this article we restrict to the cube for practical reasons.

There are many interesting questions one can ask about these convex bodies. For instance, how do they transform under birational operations? We now turn to describe a special, but important, situation where we will be able to use tools of convex optimization to say something about this question.

\section{Asymptotically log Fano/canonically polarized varieties}

Perhaps the most important line bundles in algebraic geometry are the canonical bundle of $X$, denoted $K_{X}$, and its dual, the anticanonical bundle, denoted $-K_{X}$. These two bundles give rise to two extremely important classes of varieties:

- Fano varieties are those for which $-K_{X}$ is positive [5, 10],

- Canonically polarized (general type; minimal) varieties are those for which $K_{X}$ is positive [17. (big; nef). Traditionally, algebraic geometers have been trying to classify varieties with positivity properties of $-K_{X}$ and to characterize varieties with positivity properties of $K_{X}$. The subtle difference in terminology here stems from the fact that positivity properties of $-K_{X}$ (think 'positive Ricci curvature') are rare and can sometimes be classified into a list in any given dimension, while positivity or bigness of $K_{X}$ is much more common, and hence a complete list is impossible, although one can characterize such $X$ sometimes in terms of certain traits. Be it as it may, the importance of these two classes of varieties stems from the fact that, in some very rough sense, the Minimal Model Program stipulates that all projective varieties can be built from minimal/general type and Fano pieces. Put differently, given a projective variety $K_{X}$ might not have a sign, but one should be able to perform algberaic surgeries (referred to as birational operations or birational maps) on it to eliminate the 'bad regions' of $X$ where $K_{X}$ is not well-behaved. Typically, these birational maps will make $K_{X}$ more positive (in some sense the common case, hence the terminology 'general type'), except in some rare cases when $K_{X}$ is essentially negative to begin with.

\subsection{Asymptotic logarithmic positivity associated to (anti)canonical divisors}

Thus, given the classical importance of positivity of $\pm K_{X}$, one may try to extend this to the logarithmic setting.

One may pose the following question:

Question 4.1. What are all triples $(X, D, \beta)$ such that $\beta \in \mathrm{AA}_{ \pm}\left(X,-K_{X}, D\right)$ ?

It turns out that the negative case of this question is too vast to classify, and even the positive case is out of reach unless we make some further assumptions. We now try to at 
least give some feeling for why this may be so, referring to [20, Question 8.1] for some further discussion. At the end of the day, we will distill from Question 4.1 Problem 4.6 which we will then take up in the rest of this note.

First, without some restrictions on the parameter $\beta$ Question 4.1 becomes too vast of a generalization which does not seem to be extremely useful. For this reason, 2 , we concentrate on the asymptotic logarithmic regime, where $\beta$ is required to be arbitrarily close to the origin.

Definition 4.2. [1, Definition 1.1], [20, Definition 8.13] $(X, D)$ is (strongly) asymptotically log Fano/canonically polarized if $\left(-K_{X}, D\right)$ is (strongly) asymptotically log positive/negative.

Remark 4.3. Definition 4.2 is a special case, but, in fact, the main motivation for Definition 2.1. The first, when $L=-K_{X}$, was introduced by Cheltsov and the author [1]. The second, when $L=K_{X}$, was introduced by the author [20].

Remark 4.4. When $\left(-K_{X}, D\right)$ is $\log$ positive one says $(X, D)$ is $\log$ Fano, a definition due to Maeda [15. By openess, log Fano is the most restrictive class, a subset of strongly asymptotically log Fano (ALF), itself a subset of ALF.

Remark 4.5. There is a beautiful differential geometric interpretation of Definition 4.2 in terms of Ricci curvature: $(X, D)$ is asymptotically log Fano/general type if and only if $X$ admits a Kähler metric with edge singularities of arbitrarily small angle $\beta_{i}$ along each component $D_{i}$ of the complex 'hypersurface' $D$, and moreover the Ricci curvature of this Kähler metric is positive/negative elsewhere. The only if part is an easy consequence of the definition [4, Proposition 2.2], the if part is a generalization of the Calabi-Yau theorem conjectured by Tian [21] and proved in [11, Theorem 2] when $D=D_{1}$, see also [9] for a different approach in the general case (cf. [18]). When $(X, D)$ is asymptotically log canonically polarized the statement can even be improved to the existence of a Kähler-Einstein edge metric. We refer to [20] for exposition and a survey of these and other results.

Thus, the most basic first step to understand Question 4.1 becomes the following, posed in [1].

Problem 4.6. Classify all $A L F$ pairs $(X, D)$ with $\operatorname{dim} X=2$ and $D$ having simple normal crossings.

Asymptotically log Fano varieties in dimension 2 are often referred to as asymptotically log del Pezzo surfaces. The simple normal crossings (snc) assumption is a standard one in birational geometry and is also the case that is of interest for the study of Kähler edge metrics.

\subsection{Relation to the body of ample angles}

The problem of determining whether a given pair $\left(X, D=\sum_{i=1}^{r} D_{i}\right)$ is ALF amounts to determining whether the set $\mathrm{AA}_{+}\left(X,-K_{X}, D\right)$ satisfies

$$
0 \in \overline{\mathrm{AA}_{+}\left(X,-K_{X}, D\right)} \text {. }
$$

Thus, the body of ample angles is a fundamental object in the theory of asymptotically log Fano varieties. This can also be rephrased in terms of intersection properties: there exists $\epsilon_{0}>0$ such that $\mathrm{AA}_{+}\left(X,-K_{X}, D\right) \cap B(0, \epsilon) \neq \emptyset$ for all $\epsilon \in\left(0, \epsilon_{0}\right)$, where $B(0, \epsilon)$ is the ball of radius $\epsilon$ centered at the origin in $\mathbb{R}^{r}$.

\footnotetext{
${ }^{2}$ Another important reason is that that the asymptotic logarithmic regime is closely related to understanding differential-geometric limits, as $\beta \rightarrow 0$ towards Calabi-Yau fibrations as conjectured in [1,20]
} 
If one replaces "ALF" by "strongly ALF" in Problem 4.6 the problem has been solved [1, Theorems 2.1,3.1]. However, it turns out that in the strong regime $\mathrm{AA}_{+}\left(X,-K_{X}, D\right) \subset \mathbb{R}^{4}[1$, Corollary 1.3]. In sum, the general case is out of reach using only the methods of [1]: in fact, in this note we will exhibit ALF pairs (which are necessarily not strongly ALF) for which $\mathrm{AA}_{+}\left(X,-K_{X}, D\right)$ has arbitrary large dimension and outline a strategy for classifying all ALF pairs. We hope to complete this approach in ongoing joint work with Martinez-Garcia [16].

Before describing our approach to Problem 4.6, let us pause to state an open problem concerning these bodies (for $X$ of any dimension).

Problem 4.7. How does $\mathrm{AA}_{ \pm}\left(X,-K_{X}, D\right)$ behave under birational maps of $X$ ?

\section{Convex optimization and classification in algebraic geometry}

We finally get to the heart of this note where we show how birational operations on $X$ lead to high-dimensional convex bodies.

To emphasize that we are in dimension 2 , from now on we use the notation $(S, C)$ instead of $(X, D)$. Also, since we are in the 'Fano regime' we will drop the subscript '+' and simply denote the body of ample angles

$$
\mathrm{AA}(S, C) \text {. }
$$

We denote the twisted canonical class by (recall (3.1))

$$
K_{\beta, S, C}:=K_{S}+\sum_{i=1}^{r}\left(1-\beta_{i}\right) C_{i} .
$$

The Nakai-Moishezon criterion stipulates that $\beta \in \mathrm{AA}(S, C)$ if and only if

$$
K_{\beta, S, C}^{2}>0 \text { and } K_{\beta, S, C} . Z<0 \text { for every irreducible algebraic curve } Z \text { in } X \text {. }
$$

The first is a single quadratic equation in $\beta$ while the second is a possibly infinite system of linear equations in $\beta$. We will reduce both of these to a finite system of linear equations.

To that end let us fix some $\operatorname{ALF}$ surface $(S, C)$, i.e., suppose $0 \in \overline{\mathrm{AA}(S, C)}$. We now ask:

Question 5.1. What are all ALF pairs that can be obtained as blow-ups of $(S, C)$ ?

It turns out that there are infinitely-many such pairs; the complete analysis is quite involved [16. In this article we will exhibit a particular type of (infinitely-many) such blow-ups that yields bodies of ample angles of arbitrary dimension.

\subsection{Tail blow-ups}

A snc divisor $c$ in a surface is called a chain if $c=c_{1}+\ldots+c_{r}$ with $c_{1} \cdot c_{2}=\ldots=c_{r-1} \cdot c_{r}=1$ and otherwise $c_{i} \cdot c_{j}=0$ for all $i \neq j$. In our examples each $c_{i}$ will be a smooth $\mathbb{P}^{1}$. The singular points of $c$ are the $r-1$ intersection points; all other points on $c$ are called its smooth points.

Definition 5.2. We say that $(S, C)$ is a single tail blow-up of $(s, c)$ if $S$ is the blow-up of $s$ at a smooth point of $c_{1} \cup c_{r}$, and $C=\pi^{-1}(c)$.

Note that $C$ has $r+1$ components, the 'new' component being the exceptional curve $E=\pi^{-1}(p)$ where $p \in c_{1} \cup c_{r}$. If, without loss of generality, $p \in c_{r}$ then $E . \widetilde{c}_{i}=\delta_{i r}$, so

$$
C=\widetilde{c}_{1}+\ldots+\widetilde{c}_{r}+E
$$


is still a chain.

As a very concrete example, we could take $S=\mathbb{F}_{n}$ and $C=Z_{n}+F$ (recall Example 2.3: when $n=0$ this is simply $S=\mathbb{P}^{1} \times \mathbb{P}^{1}$ and $C=\{p\} \times \mathbb{P}^{1}+\mathbb{P}^{1} \times\{q\}$, the snc divisor (with intersection point $(p, q))$ ). There are two possible single tail blow-ups: blowing-up a smooth point either on $Z_{n}$ or on $F$.

\subsection{Towards a classification of nested "tail" blow-ups}

In the notation of the previous paragraph, if $(S, C)$ is still ALF we could perform another tail blow-up, blowing up a point on $c_{1} \cup E$, and potentially repeat the process any number of times. We formalize this in a definition.

Definition 5.3. We say that $(S, C)$ is an $A L F$ tail blow-up of an $A L F$ pair $(s, c)$ if $(S, C)$ is $A L F$ and is obtained from $(s, c)$ as an iterated sequence of single tail blow-ups that result in ALF pairs in all intermediate steps.

In other words, an ALF tail blow-up is a sequence of single tail blow-ups that preserve asymptotic log positivity.

Problem 5.4. Classify all ALF tail blow-ups of ALF surfaces $\left(\mathbb{F}_{n}, c\right)$.

The following result reduces the characterization of ALF tail blow-ups to the feasibility of a certain linear program.

Define

$$
\begin{aligned}
& \operatorname{LP}(S, C):=\left\{\beta_{x} \in(0,1)^{r+x}: K_{\beta_{x}, S, C} \cdot Z<0 \quad\right. \text { for every } Z \subset S \text { such that } \pi(Z) \subset s \text { is a } \\
& \text { curve intersecting } c \text { at finitely-many points } \\
& \text { and passing through the blow-up locus, and } \\
&\left.K_{\beta_{x}, S, C} \cdot C_{i}<0, \quad i=1, \ldots, r+x .\right\}
\end{aligned}
$$

Theorem 5.5. Let $(s, c)$ be an ALF pair. An iterated sequence of $x$ single tail blow-ups $\pi: S \rightarrow s$ of $(s, c)$ is an ALF tail blow-up if only if $(i) x \leq\left(K_{s}+c\right)^{2}$, and (ii) $0 \in \overline{\mathrm{LP}(S, C)}$.

In fact, we will also show the following complementary result that shows that (essentially) the only obstacle to completely characterizing tail blow-ups are the (possibly) singular curves $Z$ passing through the blow-up locus in the definition of $\operatorname{LP}(S, C)$.

Define

$$
\widetilde{\mathrm{LP}}(S, C):=\left\{\beta_{x} \in(0,1)^{r+x}: K_{\beta_{x}, S, C} \cdot C_{i}<0, \quad i=1, \ldots, r+x\right\} .
$$

Theorem 5.6. One always has $0 \in \overline{\widetilde{\mathrm{LP}}(S, C)}$.

Before we embark on the proofs, a few remarks are in place.

Remark 5.7. Observe that $\left(K_{s}+c\right)^{2} \geq 0$. Indeed, since $(s, c)$ is ALF $-K_{s}-c$ is nef (as a limit of ample divisors), so $\left(K_{s}+c\right)^{2} \geq 0$.

Remark 5.8. The proof will demonstrate that one can drop "that result in ALF pairs in all intermediate steps" from Definition 5.3, since it follows from the fact that both $(s, c)$ and $(S, C)$ are ALF (a sort of 'interpolation' property). 
Remark 5.9. We may assume that $c$ is a connected chain of $\mathbb{P}^{1}$ 's. Indeed, when $(s, c)$ is ALF, $c$ is either a cycle or a union of disjoint chains [1, Lemma 3.5] and each component is a $\mathbb{P}^{1}$ [1, Lemmas 3.2]. The former is irrelevant for us since there are no tails. For the latter, we may assume that $c$ is connected (i.e., one chain) since the only disconnected case, according to the classification results [1, Theorems 2.1,3.1], is $\left(\mathbb{F}_{n}, c_{1}+c_{2}\right)$ with $c_{1}=Z_{n}$ and $c_{2} \in\left|Z_{n}+n F\right|$ and then $\left(K_{\mathbb{F}_{n}}+c_{1}+c_{2}\right)^{2}=0$ so no tail blow-ups are allowed by Remark 5.16. To see that, let $c_{1} \in\left|a Z_{n}+b F\right|$ and $c_{2} \in\left|A Z_{n}+B F\right|$. Since $c_{1}, c_{2}$ are effective, $b \geq n a, B \geq n A$. By assumption $c_{1} \cap c_{2}=\emptyset$ so $0=c_{1} \cdot c_{2}=-n a A+a B+b A$, i.e., $b A=a(n A-B)$. Since the right hand side is nonpositive and the left hand side is nonnegative they must both be zero, leading to $b=0, B=n A$ ( $A=0$ is impossible since it would force $B=0$, and $a=0$ is excluded by $b=0)$. Thus we see $c_{1} \in\left|a Z_{n}\right|, c_{2} \in\left|A\left(Z_{n}+n F\right)\right|$. There are no smooth irreducible representatives of $\left|a Z_{n}\right|$ unless $a=1$ and similarly for $\left|A\left(Z_{n}+n F\right)\right|$ unless $A=1$.

Remark 5.10. Theorems 5.5 and 5.6 are mainly given for illustrative reasons, i.e., to explicitly show how tools of convex programming can be used in this context. As we show in a forthcoming extensive, but unfortunately long and tedious, classification work [16 the case of tail blow-ups is in fact the "worst" in terms of preserving asymptotic log positivity. We will give there a classification of asymptotically log del Pezzo surfaces that completely avoids tail blow-ups since condition (ii) in Theorem 5.5] is difficult to control, in general. Thus, the present note and [16] are somewhat complementary. It is still an interesting open problem to classify all ALF tail blow-ups.

\subsection{The set-up}

We start with an ALF pair $\left(s, c=c_{1}+\ldots+c_{r}\right)$ and perform $v+h$ single tail blow-ups of which

$$
h \text { ('högra') tail blow-ups on the "right tail" } c_{r}
$$

with associated blow-down map

$$
\pi_{H}=\pi_{1} \circ \cdots \circ \pi_{h}
$$

and exceptional curves

$$
\operatorname{exc}\left(\pi_{i}\right)=H_{i}, \quad i=1, \ldots, h
$$

and of which

$$
v \text { ('vänster') tail blow-ups on the "left tail" } c_{1}
$$

with blow-down map

$$
\pi_{V}=\pi_{h+1} \circ \cdots \circ \pi_{h+v}
$$

and exceptional curves

$$
\operatorname{exc}\left(\pi_{h+j}\right)=V_{j}, \quad i=1, \ldots, v,
$$

with new angles $\eta \in(0,1)^{h}$ and $\nu \in(0,1)^{v}$, respectively. Finally, we set

$$
\eta_{0}:=\beta_{r}, \quad \nu_{0}:=\beta_{1} .
$$

An induction argument shows:

Lemma 5.11. With the notation (5.4) (5.10), if $v, h>0$,

$$
\begin{aligned}
& -K_{(\beta, \nu, \eta), S,\left(\pi_{H} \circ \pi_{V}\right)^{-1}(c)}= \\
& -\pi_{V}^{*} \pi_{H}^{*} K_{\beta, s, c}-\sum_{i=1}^{h}\left(1-\eta_{i}+\eta_{i-1}\right) \pi_{V}^{*} \pi_{h}^{*} \cdots \pi_{i+1}^{*} H_{i}-\sum_{j=1}^{v}\left(1-\nu_{j}+\nu_{j-1}\right) \pi_{h+v}^{*} \cdots \pi_{h+1+j}^{*} V_{j} .
\end{aligned}
$$


If $v=0$,

$$
-K_{(\beta, \eta, \nu), S, \pi_{H}^{-1}(c)}=-\pi_{H}^{*} K_{\beta, s, c}-\sum_{i=1}^{h}\left(1-\eta_{i}+\eta_{i-1}\right) \pi_{h}^{*} \cdots \pi_{i+1}^{*} H_{i} .
$$

If $h=0$,

$$
-K_{(\beta, \eta, \nu), S, \pi_{V}^{-1}(c)}=-\pi_{V}^{*} \pi_{H}^{*} K_{\beta, s, c}-\sum_{j=1}^{v}\left(1-\nu_{j}+\nu_{j-1}\right) \pi_{v+h}^{*} \cdots \pi_{h+1+j}^{*} V_{j} .
$$

Before giving the proof, let us recall two elementary facts about blow-ups. Let $\pi: S_{2} \rightarrow S_{1}$ be the blow-up at a smooth point $p$ on a surface $S_{1}$. Then,

$$
K_{S_{2}}=\pi^{*} K_{S_{1}}+E
$$

where $E=\pi^{-1}(p)$ [7, p. 187], and for every divisor $F \subset S_{1}$,

$$
\widetilde{F}= \begin{cases}\pi^{*} F, & \text { if } p \notin F, \\ \pi^{*} F-E, & \text { otherwise. }\end{cases}
$$

Proof. Using (5.14), if $v=0$,

$$
K_{S}=\pi_{h}^{*}\left(\pi_{h-1}^{*}\left(\cdots\left(\pi_{1}^{*}\left(K_{s}+H_{1}\right)+H_{2}\right)+\ldots+H_{h-2}\right)+H_{h-1}\right)+H_{h} .
$$

Similarly, if $h=0$,

$$
K_{S}=\pi_{v}^{*}\left(\pi_{v-1}^{*}\left(\cdots\left(\pi_{1}^{*}\left(K_{s}+V_{1}\right)+V_{2}\right)+\ldots+V_{v-2}\right)+V_{v-1}\right)+V_{v} .
$$

If $v, h>0$,

$$
\begin{aligned}
& K_{S}=\pi_{v+h}^{*}\left(\pi_{v+h-1}^{*}(\right. \cdots\left(\pi _ { h + 1 } ^ { * } \left(\pi _ { h } ^ { * } \left(\cdots\left(\pi_{1}^{*}\left(K_{s}+H_{1}\right)+H_{2}\right)\right.\right.\right. \\
&\left.\left.\left.\left.+\ldots+)+H_{h}\right)+V_{1}\right)+\ldots+V_{v-2}\right)+V_{v-1}\right)+V_{v} .
\end{aligned}
$$

Using (5.15) and (5.10), if $v=0$,

$$
\begin{aligned}
\sum_{i=1}^{r+h}\left(1-\beta_{i}\right) C_{i}= & \sum_{i=1}^{r-1}\left(1-\beta_{i}\right) \pi_{H}^{*} c_{i}+\left(1-\beta_{r}\right) \pi_{h}^{*} \cdots \pi_{2}^{*}\left(\pi_{1}^{*} c_{r}-H_{1}\right) \\
& +\left(1-\eta_{1}\right) \pi_{h}^{*} \cdots \pi_{3}^{*}\left(\pi_{2}^{*} H_{1}-H_{2}\right)+\ldots+\left(1-\eta_{h-1}\right)\left(\pi_{H}^{*} H_{h-1}-H_{h}\right) \\
& +\left(1-\eta_{h}\right) H_{h} \\
= & \sum_{i=1}^{r}\left(1-\beta_{i}\right) \pi_{H}^{*} c_{i}+\sum_{i=1}^{h}\left(\eta_{i-1}-\eta_{i}\right) \pi_{h}^{*} \cdots \pi_{i+1}^{*} H_{i}
\end{aligned}
$$


if $h=0$,

$$
\begin{aligned}
\sum_{i=1}^{r+v}\left(1-\beta_{i}\right) C_{i}=( & \left.-\beta_{1}\right) \pi_{v+h}^{*} \cdots \pi_{h+2}^{*}\left(\pi_{h+1}^{*} c_{1}-V_{1}\right)+\sum_{i=2}^{r}\left(1-\beta_{i}\right) \pi_{V}^{*} c_{i} \\
& +\left(1-\nu_{1}\right) \pi_{v+h}^{*} \cdots \pi_{h+2}^{*}\left(\pi_{h+1}^{*} V_{1}-V_{2}\right)+\ldots+\left(1-\nu_{v-1}\right)\left(\pi_{v+h}^{*} V_{v-1}-V_{v}\right) \\
& \quad+\left(1-\nu_{v}\right) V_{v} \\
= & \sum_{i=1}^{r}\left(1-\beta_{i}\right) \pi_{V}^{*} c_{i}+\sum_{i=1}^{v}\left(\nu_{i-1}-\nu_{i}\right) \pi_{v}^{*} \cdots \pi_{i+1}^{*} V_{i},
\end{aligned}
$$

and if $v, h>0$,

$$
\begin{aligned}
\sum_{i=1}^{r+v+h}\left(1-\beta_{i}\right) C_{i}=( & \left.-\beta_{1}\right) \pi_{v+h}^{*} \cdots \pi_{h+2}^{*}\left(\pi_{h+1}^{*} \pi_{H}^{*} c_{1}-V_{1}\right) \\
& +\sum_{i=2}^{r-1}\left(1-\beta_{i}\right) \pi_{V}^{*} \pi_{H}^{*} c_{i}+\left(1-\beta_{r}\right) \pi_{V}^{*} \pi_{h}^{*} \cdots \pi_{2}^{*}\left(\pi_{1}^{*} c_{r}-H_{1}\right) \\
& +\left(1-\eta_{1}\right) \pi_{V}^{*} \pi_{h}^{*} \cdots \pi_{3}^{*}\left(\pi_{2}^{*} H_{1}-H_{2}\right)+\ldots+\left(1-\eta_{h-1}\right) \pi_{V}^{*}\left(\pi_{h}^{*} H_{h-1}-H_{h}\right) \\
& +\left(1-\eta_{h}\right) \pi_{V}^{*} H_{h} \\
& +\left(1-\nu_{1}\right) \pi_{v+h}^{*} \cdots \pi_{h+2}^{*}\left(\pi_{h+1}^{*} V_{1}-V_{2}\right)+\ldots+\left(1-\nu_{v-1}\right)\left(\pi_{v+h}^{*} V_{v-1}-V_{v}\right) \\
& +\left(1-\nu_{v}\right) V_{v} \\
=\sum_{i=1}^{r}(1- & \left.\beta_{i}\right) \pi_{V}^{*} \pi_{H}^{*} c_{i}+\sum_{i=1}^{h}\left(\eta_{i-1}-\eta_{i}\right) \pi_{V}^{*} \pi_{h}^{*} \cdots \pi_{i+1}^{*} H_{i}+\sum_{i=1}^{v}\left(\nu_{i-1}-\nu_{i}\right) \pi_{v}^{*} \cdots \pi_{i+1}^{*} V_{i} .
\end{aligned}
$$

Thus, (5.18) and (5.21) imply (5.11), (5.16) and (5.19) imply (5.12), and (5.17) and (5.20) imply (5.13).

Remark 5.12. In principle, as we will see below, the blow-ups on the left and on the right do not interact.

\subsection{The easy direction and the sub-critical case}

We start with a simple observation. The easy direction of Theorem 5.5 is contained in the next lemma:

Lemma 5.13. Let $(s, c)$ be an ALF pair. Let $(S, C)$ be obtained from $(s, c)$ via an iterated sequence of $x$ single tail blow-ups of $(s, c)$. Then $(S, C)$ is not $A L F$ if $x>\left(K_{s}+c\right)^{2}$.

Proof. If $c$ does not contain a tail, there is nothing to prove. By Remark 5.16, we may assume that $c$ is a single chain. Let $\pi: S \rightarrow s$ denote the blow-up of a point on a tail $c_{r}$ with exceptional curve $E=: C_{r+1}$. Then,

$$
\begin{aligned}
-K_{\left(\beta, \beta_{r+1}\right), S, C+E} & =-\pi^{*} K_{s}-E-\sum_{i=1}^{r}\left(1-\beta_{i}\right) \widetilde{c}_{i}-\left(1-\beta_{r+1}\right) E \\
& =-\pi^{*} K_{s}-E-\sum_{i=1}^{r-1}\left(1-\beta_{i}\right) \pi^{*} c_{i}-\left(1-\beta_{r}\right)\left(\pi^{*} c_{r}-E\right)-\left(1-\beta_{r+1}\right) E \\
& =-\pi^{*} K_{\beta, s, c}-\left(1+\beta_{r}-\beta_{r+1}\right) E .
\end{aligned}
$$


In particular, since $E^{2}=-1, K_{(0,0), S, C+E}^{2}=K_{0, s, c}^{2}-1$. An induction (or directly using Lemma 5.11) thus shows that $\left(K_{S}+C\right)^{2}=\left(K_{s}+c\right)^{2}-x$, which shows that $-K_{S}-C$ cannot be nef if $x>\left(K_{s}+c\right)^{2}$, so $(S, C)$ cannot be ALF, by Remark 5.7.

\subsection{Dealing with the quadratic constraint and the critical case}

Let

$$
\beta_{x}=\left(\beta, \beta_{r+1}, \ldots, \beta_{r+x}\right) \in \mathbb{R}^{r+x} .
$$

The proof of Lemma 5.13 also shows that

$$
K_{\beta_{x}, S, C}^{2}=K_{\beta, s, c}^{2}-x+f\left(\beta_{x}\right),
$$

where $f: \mathbb{R}^{r+x} \rightarrow \mathbb{R}$ is a quadratic polynomial with no constant term and whose coefficients are integers bounded by a constant depending only on $r+x$. Thus, we also obtain some information regarding the converse to Lemma 5.13 .

Corollary 5.14. Let $(s, c)$ be an $A L F$ pair. Let $(S, C)$ be obtained from $(s, c)$ via an iterated sequence of $x$ single tail blow-ups of $(s, c)$. Then $K_{\beta, S, C}^{2}>0$ for all sufficiently small (depending only on $r, x$, hence only on $r, s, c) \beta_{x} \in \mathbb{R}^{r+x}$ if $x<\left(K_{s}+c\right)^{2}$.

This corollary is useful since it implies the quadratic inequality in (5.1) can be completely ignored except, perhaps, in the borderline case $x=\left(K_{s}+c\right)^{2}$.

The next result treats precisely that borderline case:

Proposition 5.15. Let $(s, c)$ be an $A L F$ pair. Let $(S, C)$ be obtained from $(s, c)$ via an iterated sequence of $x:=\left(K_{s}+c\right)^{2}$ single tail blow-ups of $(s, c)$. Then

$$
K_{\beta, S, C}^{2}=f\left(\beta_{x}\right)
$$

where $f: \mathbb{R}^{r+x} \rightarrow \mathbb{R}$ is a quadratic polynomial with no constant term and whose coefficients are integers bounded by a constant depending only on $r+x$, and moreover it contains linear terms with positive coefficients and no linear terms with negative coefficients. In particular, $K_{\beta, S, C}^{2}>0$ for all sufficiently small (depending only on $r, x$, hence only on $\left.r, s, c\right) \beta_{x} \in(0,1)^{r+x}$.

Remark 5.16. The key for later will be (5.22) rather than the conclusion about $K_{\beta, S, C}^{2}>0$ for all sufficiently small angles. In fact, the latter conclusion (at the end of Proposition [5.15) is not precise enough to conclude that the quadratic inequality in (5.1) can be ignored as one needs that it holds simultaneously with the intersection inequalities of (5.1). The exact form of (5.22) implies that (5.22) can be satisfied together with any linear constraints on $\beta_{x}$, which will be the key, and the reason that, ultimately, the quadratic inequality in (5.1) can be ignored.

Proof. We use the notation of $\$ 5.3$. We wish to show that

$$
K_{(\beta, \delta, \gamma), S,\left(\pi_{H} \circ \pi_{V}\right)^{-1}(c)}^{2}>0, \quad \text { for some small }(\beta, \delta, \gamma) \in(0,1)^{r+h+v}
$$


$\left(\right.$ recall $\left.x=h+v=\left(K_{s}+c\right)^{2}\right)$. We compute,

$$
\begin{aligned}
K_{(\beta, \delta, \gamma), S,\left(\pi_{H} \circ \pi_{V}\right)^{-1}(c)=}^{2} K_{\beta, s, c}-\sum_{i=1}^{h}\left(1-\delta_{i}+\delta_{i-1}\right)^{2}-\sum_{j=1}^{v}\left(1-\gamma_{j}+\gamma_{j-1}\right)^{2} \\
=\left(K_{s}+c\right)^{2}-2 \sum_{i=1}^{r} \beta_{i} c_{i} \cdot\left(K_{s}+c\right)+\sum_{i=1}^{r} \beta_{i}^{2} c_{i}^{2} \\
\quad-h+2 \sum_{i=1}^{h} \delta_{i}-2 \sum_{i=1}^{h} \delta_{i-1}-v+2 \sum_{j=1}^{v} \gamma_{j}-2 \sum_{j=1}^{v} \gamma_{j-1} \\
\quad-\sum_{i=1}^{h}\left(\delta_{i}-\delta_{i-1}\right)^{2}-\sum_{j=1}^{v}\left(\gamma_{j}-\gamma_{j-1}\right)^{2} \\
=-2 \sum_{i=1}^{r} \beta_{i} c_{i} .\left(K_{s}+c\right)+2 \delta_{h}-2 \beta_{r}+2 \gamma_{v}-2 \beta_{1}-O\left(\beta^{2}, \delta^{2}, \gamma^{2}\right) \\
=2 \beta_{1}+2 \beta_{r}+2 \delta_{h}-2 \beta_{r}+2 \gamma_{v}-2 \beta_{1}-O\left(\beta^{2}, \delta^{2}, \gamma^{2}\right) \\
=2 \delta_{h}+2 \gamma_{v}-O\left(\beta^{2}, \delta^{2}, \gamma^{2}\right),
\end{aligned}
$$

since, by Remark 5.9, all $c_{i}$ are smooth rational curves and $c$ is a single chain, so by adjunction

$$
c_{i} \cdot\left(K_{s}+c\right)= \begin{cases}c_{i} \cdot\left(K_{s}+c_{i}\right)+c_{i} \cdot c_{i-1}+c_{i} \cdot c_{i+1}=-2+1+1=0, & \text { if } i=2, \ldots, r-1, \\ c_{r} \cdot\left(K_{s}+c_{r}\right)+c_{r} \cdot c_{r-1}=-2+1=-1, & \text { if } i=r, \\ c_{1} \cdot\left(K_{s}+c_{1}\right)+c_{1} \cdot c_{2}=-2+1=-1, & \text { if } i=1 .\end{cases}
$$

This is clearly positive for $(\beta, \delta, \gamma)=\epsilon(1, \ldots, 1)$ for $\epsilon$ small enough. This proves the Proposition.

Remark 5.17. As alluded to in the remark preceeding the proof, one indeed can make $2 \delta_{h}+$ $2 \gamma_{v}-O\left(\beta^{2}, \delta^{2}, \gamma^{2}\right)$ positive under any linear constraints on $\beta, \delta, \gamma$ without imposing any new linear constraints as the coefficients of the only non-zero linear terms are positive.

\subsection{Proof of Theorem 5.5}

First, suppose either (i) or (ii) does not hold. If (i) fails then Lemma 5.13 shows that $(S, C)$ is not ALF. If (ii) fails then $(S, C)$ is not ALF by Definition 4.2 .

Second, if both (i) and (ii) hold then Corollary 5.14, Proposition 5.15, and the NakaiMoishezon criterion show that $(S, C)$ is ALF if and only if $K_{\beta_{x}, S, C} . Z<0$ for every irreducible curve $Z \subset S$. Naturally, we distinguish between three types of curves $Z$ :

(a) $\pi(Z)$ does not pass through the blow-up locus,

(b) $\pi(Z)$ is contained in the blow-up locus,

(c) $\pi(Z)$ is a curve passing through the blow-up locus.

Curves of type (a) can be ignored: Indeed, then $\pi(Z)$ is a curve in $s$ and $Z=\pi^{*} \pi(Z)$ (hence, does not intersect any of the exceptional curves) so by Lemma 5.11.

$$
K_{\beta, S, C} \cdot Z=\pi^{*} K_{\left(\beta_{1}, \ldots, \beta_{r}\right), s, c} \cdot \pi^{*} \pi(Z)=K_{\left(\beta_{1}, \ldots, \beta_{r}\right), s, c} \cdot \pi(Z) .
$$

As $(s, c)$ is $\mathrm{ALF}$, this intersection number is negative. 
Next, curves of type (c) are covered by condition (ii) by the definition of $\operatorname{LP}(S, C)$. Finally, since curves of type (b) are, by definition of the tail blow-up, components of the new boundary $C$, hence there are at most $x+2$ (i.e., finitely-many) of them, certainly contained in the finitely-many inequalities:

$$
K_{\beta_{x}, S, C} \cdot C_{i}<0, \quad i=1, \ldots, r+x,
$$

which are once again covered by the definition of $\operatorname{LP}(S, C)$. This concludes the proof of Theorem 5.5 .

\subsection{Reduction of the linear intersection constraints}

In this subsection we explain how to essentially further reduce the linear intersection constraints, i.e., we prove Theorem 5.6. To that purpose, we show that curves of type (b) can be handled directly. This shows that the only potential loss of asymptotic logarithmic positivity occurs from curves of type (c) (observe that as in the previous subsection, curves of type (a) can be ignored).

Proof of Theorem 5.6. It suffices to check that the system of $2 r+2 x$ inequalities

$$
\begin{aligned}
K_{\beta_{x}, S, C \cdot C} C_{i}<0, & i=1, \ldots, r+x, \\
\beta_{i}>0, & i=1, \ldots, r+x,
\end{aligned}
$$

admit a solution along some ray emanating from the origin in $\mathbb{R}^{r+x}$.

Let us first write these inequalities carefully and by doing so eliminate some unnecessary ones.

Using Lemma 5.11 we compute, starting with the tails, which turn out to pose no constraints, to wit,

$$
\begin{aligned}
-K_{(\beta, \delta, \gamma), S,\left(\pi_{H} \circ \pi_{V}\right)^{-1}(c)} \cdot V_{v} & =1-\gamma_{v}+\gamma_{v-1}>0, \\
-K_{(\beta, \delta, \gamma), S,\left(\pi_{H} \circ \pi_{V}\right)^{-1}(c)} \cdot \pi_{V}^{*} H_{h} & =1-\delta_{h}+\delta_{h-1}>0 .
\end{aligned}
$$

Next, we intersect with the other new boundary curves (if $h, v>0$ there are $h+v-2$ such, if $h=0$ there are $v-1 \mathrm{such}$, if $v=0$ there are $h-1 \mathrm{such})$,

$$
\begin{aligned}
-K_{(\beta, \delta, \gamma), S,\left(\pi_{H} \circ \pi_{V}\right)^{-1}(c)} \cdot \pi_{h+v}^{*} \cdots \pi_{h+j+1}^{*}\left(\pi_{h+j}^{*} V_{j-1}-V_{j}\right) & =\left(1-\gamma_{j-1}+\gamma_{j-2}\right)-\left(1-\gamma_{j}+\gamma_{j-1}\right) \\
& =\gamma_{j}-2 \gamma_{j-1}+\gamma_{j-2}, \quad j=2, \ldots, v . \\
-K_{(\beta, \delta, \gamma), S,\left(\pi_{H} \circ \pi_{V}\right)^{-1}(c)} \cdot \pi_{V}^{*} \pi_{h}^{*} \cdots \pi_{i+1}^{*}\left(\pi_{i}^{*} H_{i-1}-H_{i}\right) & =\left(1-\delta_{i-1}+\delta_{i-2}\right)-\left(1-\delta_{i}+\delta_{i-1}\right) \\
& =\delta_{i}-2 \delta_{i-1}+\delta_{i-2}, \quad i=2, \ldots, h .
\end{aligned}
$$

Finally, we intersect with the two 'old tails' (or only one if $\min \{h, v\}=0$ ), and use (5.25),

$$
\begin{aligned}
-K_{(\beta, \delta, \gamma), S,\left(\pi_{H} \circ \pi_{V}\right)^{-1}(c)} \cdot \pi_{h+v}^{*} \cdots \pi_{h+2}^{*}\left(\pi_{h+1}^{*} \pi_{H}^{*} c_{1}-V_{1}\right) & =-K_{\beta, s, c} \cdot c_{1}-\left(1-\gamma_{1}+\beta_{1}\right) \\
& =1+\beta_{1} c_{1}^{2}-\left(1-\gamma_{1}+\beta_{1}\right) \\
& =\gamma_{1}+\left(c_{1}^{2}-1\right) \beta_{1} \\
-K_{(\beta, \delta, \gamma), S,\left(\pi_{H} \circ \pi_{V}\right)^{-1}(c)} . \pi_{V}^{*} \pi_{h}^{*} \cdots \pi_{2}^{*}\left(\pi_{1}^{*} c_{r}-H_{1}\right) & =-K_{\beta, s, c} \cdot c_{r}-\left(1-\delta_{1}+\beta_{r}\right) \\
& =1+\beta_{r} c_{r}^{2}-\left(1-\delta_{1}+\beta_{r}\right) \\
& =\delta_{1}+\left(c_{r}^{2}-1\right) \beta_{r} .
\end{aligned}
$$


Equations (5.28)-(5.29) are $h+v$ linear equations that together with the $r+h+v$ constraints

$$
\beta_{x}=(\beta, \delta, \gamma) \in \mathbb{R}_{+}^{r+h+v}
$$

can be encoded by a $(r+h+v)$-by- $(r+2 h+2 v)$ matrix inequality:

$$
(\beta, \delta, \gamma) \operatorname{LP}\left(S,\left(\pi_{H} \circ \pi_{V}\right)^{-1}(c)\right)>0,
$$

where the inequality symbol means that each component of the vector is positive (typical notation in linear optimization, see, e.g., [2]) with

$$
\operatorname{LP}\left(S,\left(\pi_{H} \circ \pi_{V}\right)^{-1}(c)\right):= \begin{cases}\left(\begin{array}{llll}
v_{r} & v_{1} & T & I_{r+h+v}
\end{array}\right) & \text { if } h, v>0, \\
\left(\begin{array}{lll}
v_{r} & T & I_{r+h}
\end{array}\right) & \text { if } h>0, v=0, \\
\left(\begin{array}{lll}
v_{1} & T & I_{r+v}
\end{array}\right) & \text { if } h=0, v>0,\end{cases}
$$

where

$$
\begin{aligned}
& v_{r}=(\overbrace{0, \ldots, 0}^{r-1}, c_{r}^{2}-1,1, \overbrace{0, \ldots, 0}^{h-1}, \overbrace{0, \ldots, 0}^{v})^{T} \in \mathbb{Z}^{h+v+r}, \\
& v_{1}=(c_{1}^{2}-1, \overbrace{0, \ldots, 0}^{r-1}, \overbrace{0, \ldots, 0}^{h}, 1, \overbrace{0, \ldots, 0}^{v-1})^{T} \in \mathbb{Z}^{h+v+r}, \\
& T=\left\{\begin{array}{l}
\left(\begin{array}{cc}
T_{r} & T_{1} \\
T_{h} & 0_{h, v-1} \\
0_{v, h-1} & T_{v}
\end{array}\right) \in \mathrm{Mat}_{r+h+v, h+v-2}, \text { if } h, v>0 \\
\left(\begin{array}{c}
T_{r} \\
T_{h}
\end{array}\right) \in \mathrm{Mat}_{r+h, h-1}, \text { if } h>0, v=0 \\
\left(\begin{array}{c}
T_{1} \\
T_{v}
\end{array}\right) \in \mathrm{Mat}_{r+v, v-1}, \text { if } h=0, v>0
\end{array}\right.
\end{aligned}
$$

with

$$
\begin{aligned}
T_{r} & =\left(\begin{array}{cccc}
0 & 0 & \ldots & 0 \\
\vdots & \vdots & \ldots & \vdots \\
0 & 0 & \ldots & 0 \\
1 & 0 & \ldots & 0
\end{array}\right) \in \operatorname{Mat}_{r, h-1}, \quad T_{1}=\left(\begin{array}{cccc}
1 & 0 & \ldots & 0 \\
0 & 0 & \ldots & 0 \\
\vdots & \vdots & \ldots & \vdots \\
0 & 0 & \ldots & 0
\end{array}\right) \in \text { Mat }_{r, v-1}, \\
T_{h} & =\left(\begin{array}{cccc}
-2 & 1 & \ldots & 0 \\
1 & -2 & \ldots & 0 \\
0 & 1 & \ldots & 0 \\
\vdots & \vdots & \ldots & \vdots \\
0 & \ldots & 0 & 1 \\
0 & 0 & \ldots & -2 \\
0 & \ldots & 0 & 1
\end{array}\right) \in \operatorname{Mat}_{h, h-1}, \quad T_{v}=\left(\begin{array}{cccc}
-2 & 1 & \ldots & 0 \\
1 & -2 & \ldots & 0 \\
0 & 1 & \ldots & 0 \\
\vdots & \vdots & \ldots & \vdots \\
0 & \ldots & 0 & 1 \\
0 & 0 & \ldots & -2 \\
0 & \ldots & 0 & 1
\end{array}\right) \in \text { Mat }_{v, v-1},
\end{aligned}
$$

(here, we use the convention that $T_{r}$ and $T_{h}$ are the empty matrix if $h<2$ and similarly for $T_{1}$ and $T_{v}$ if $v<2$ ). 
By Gordan's Theorem [2, p. 136], the inequalities (5.30) hold if and only if the only solution $y \in \mathbb{R}_{+}^{r+2 h+2 v}$ to

$$
\operatorname{LP}\left(S,\left(\pi_{H} \circ \pi_{V}\right)^{-1}(c)\right) y=0
$$

is $y=0 \in \mathbb{R}_{+}^{r+2 h+2 v}$. We treat first the (easy) cases

$$
(h, v) \in\{(1,0),(0,1),(2,0),(0,2),(1,1),(2,1),(1,2)\}
$$

separately.

The case $(1,0)$ imposes only the inequality $\delta_{1}+\left(c_{r}^{2}-1\right) \beta_{r}>0$ which is feasible. Similarly, the case $(0,1)$ imposes only $\gamma_{1}+\left(c_{1}^{2}-1\right) \beta_{1}>0$. The case $(1,1)$ imposes both of these inequalities, but they are independent, hence feasible.

The case $(2,0)$ imposes the inequalities

$$
\delta_{1}+\left(c_{r}^{2}-1\right) \beta_{r}>0, \quad \delta_{2}-2 \delta_{1}+\beta_{r}>0,
$$

which are equivalent via a Fourier-Motzkin elimination [2, §4.4] to $\delta_{2}+\beta_{r}>2\left(1-c_{r}^{2}\right) \beta_{r}$, i.e., $\delta_{2}>\left(1-2 c_{r}^{2}\right) \beta_{r}$, which is feasible. The case $(0,2)$ is handled similarly. The case $(2,2)$ is feasible for the same reasons: both sets of inequalities are feasible and independent. The case $(2,1)$ (and similarly $(1,2))$ also follows since it imposes the inequalities (5.31) in addition to the independent inequality $\gamma_{1}+\left(c_{1}^{2}-1\right) \beta_{1}>0$, thus these are feasible. This idea of independence will also be useful in the general case below.

Let us turn to the general case, i.e., suppose $h, v \geq 2$. First, the $r+h$-th row of $\operatorname{LP}\left(S,\left(\pi_{H} \circ\right.\right.$ $\left.\left.\pi_{V}\right)^{-1}(c)\right)$ is

$$
\overbrace{0, \ldots, 0}^{h}, 1, \overbrace{0, \ldots, 0}^{v-1+r+h-1}, 1, \overbrace{0, \ldots, 0}^{v})
$$

This implies $y_{h+1}=y_{r+2 h+v-1}=0$. If $h=2$ this implies $y_{1}=y_{r+2 h+v-2}=0$; if $h>3$ this implies $y_{h}=y_{r+2 h+2 v-2}=0$ (the -2 in the $(h+1)$-th spot in that row is taken care of by the fact $y_{h+1}=0$ from the previous step), and inductively we obtain $y_{h+1-i}=y_{r+2 h+2 v-i}=0, i=$ $1, \ldots, h-2$, and finally $y_{1}=y_{r+h+2 v+1}=0$. Altogether, we have shown $2 h$ of the $y_{i}$ 's are zero.

Second, the $r+h+v$-th (last) row is

$$
(\overbrace{0, \ldots, 0}^{h+v-1}, 1, \overbrace{0, \ldots, 0}^{r+h+v-1}, 1)
$$

This implies $y_{h+v}=y_{r+2 h+2 v}=0$. If $v>2$ this implies $y_{h+v-1}=y_{r+2 h+2 v-1}=0$, and inductively we obtain $y_{h+v-i}=y_{r+2 h+2 v-i}=0, i=1, \ldots, v-2$, and finally $y_{2}=y_{r+2 h+v+1}=0$. In this step we have shown $2 v$ of the $y_{i}$ 's are zero.

So far we have shown $2 h+2 v$ of the $y_{i}$ 's are zero using the last $2 h+2 v$ rows.

Finally, we consider the first $r$ rows. There are two special rows with possibly positive coefficients $c_{r}^{2}-1$ and $c_{1}^{2}-1$, however the corresponding $y_{1}$ and $y_{2}$ are zero, so as we have the full rank and identity matrix $I$ (with nonnegative coefficients) in $\operatorname{LP}\left(S,\left(\pi_{H} \circ \pi_{V}\right)^{-1}(c)\right)$ it follows that the remaining $r$ variables $y_{i}$ are zero, concluding the proof of Theorem 5.6 .

\section{References}

[1] I.A. Cheltsov, Y.A. Rubinstein, Asymptotically log Fano varieties, Adv. Math. 285 (2015), 1241-1300.

[2] G.B. Dantzig, Linear programming and extensions, Princeton Univ. Press, 1963. 
[3] T. Delzant, Hamiltoniens périodiques et images convexes de l'application moment, Bull. Soc. Math. France 116 (1988), 315-339.

[4] L. Di Cerbo, On Kähler-Einstein surfaces with edge singularities, J. Geom. Phys. 86 (2014), 414-421.

[5] G. Fano, Sulle varietà algebriche a tre dimensioni a curve-sezioni canoniche, Comment. Math. Helv. 14 (1941-1942), 23-64.

[6] W. Fulton, Introduction to toric varieties, Princeton University Press, 1993.

[7] P. Griffiths, J. Harris, Principles of algebraic geometry, Wiley-Interscience, 1978.

[8] M. Gromov, Convex set and Kähler manifolds, in: Advances in differential geometry and topology, World Sci. Publ., 1990, pp. 1-38.

[9] H. Guenancia, M. Păun, Conic singularities metrics with prescribed Ricci curvature: The case of general cone angles along normal crossing divisors, J. Diff. Geom. 103 (2016), $15-57$.

[10] V.A. Iskovskikh, Yu.G. Prokhorov, Fano varieties, in: Algebraic geometry, V, Springer, 1999, pp. 1-247.

[11] T. Jeffres, R. Mazzeo, Y.A. Rubinstein, Kähler-Einstein metrics with edge singularities, (with an appendix by C. Li and Y.A. Rubinstein), Annals of Math. 183 (2016), 95-176.

[12] K. Kaveh, A. Khovanskii, Algebraic equations and convex bodies, in: Perspectives in analysis, geometry, and topology, Birkhäuser, 2012, pp. 263-282.

[13] R. Lazarsfeld, Positivity in algebraic geometry, I, II, Springer, 2004.

[14] R. Lazarsfeld, M. Mustata, Convex bodies associated to linear series, Ann. Sci. Éc. Norm. Supér. (4) 42 (2009), 783-835.

[15] H. Maeda, Classification of logarithmic threefolds, Compositio Math. 57 (1986), 81-125.

[16] J. Martinez-Garcia, Y.A. Rubinstein, Asymptotically log del Pezzo surfaces, work in progress.

[17] T. Matsusaka, On canonically polarized varieties, in: 1969 Algebraic Geometry (Internat. Colloq., Tata Inst. Fund. Res., Bombay, 1968), Oxford Univ. Press, pp. 265-306.

[18] R. Mazzeo, Y.A. Rubinstein, The Ricci continuity method for the complex Monge-Ampère equation, with applications to Kähler-Einstein edge metrics, C. R. Math. Acad. Sci. Paris 350 (2012), 693-697.

[19] T. Oda, Convex bodies and algebraic geometry. An introduction to the theory of toric varieties, Springer, 1988.

[20] Y.A. Rubinstein, Smooth and singular Kähler-Einstein metrics, in: Geometric and Spectral Analysis (P. Albin et al., Eds.), Contemp. Math. 630, Amer. Math. Soc. and Centre de Recherches Mathématiques, 2014, 45-138.

[21] G. Tian, Kähler-Einstein metrics on algebraic manifolds, Lecture Notes in Math. 1646 (1996), 143-185.

\author{
UNIVERSITY OF MARYLAND \\ yanir@umd.edu
}

